\title{
Ocorrência de quedas e seu contexto num seguimento de dois anos em idosos institucionalizados
}

\author{
The occurrence of falls and their context in a two-year follow up of institutionalized elderly persons
}

Ocurrencia de caídas y su contexto en un seguimiento de dos años a ancianos institucionalizados

Ruth Losada de Menezes ${ }^{1}$, Maria Márcia Bachion²

\section{RESUMO}

Este estudo teve como objetivo identificar, em idosos institucionalizados, a ocorrência de quedas e caracterizar o seu contexto. Trata-se de estudo descritivo-exploratório, com abordagem quantitativa, realizado em cinco instituições de longa permanência para idosos no município de Goiânia-GO. Foi aplicado um questionário que abordava informações a respeito da ocorrência de quedas no seguimento 2005-2007 e seu contexto. Participaram do estudo 59 idosos que atenderam aos critérios de seleção. Os resultados mostraram ocorrência de $54,2 \%$ de prevalência de quedas no período. Quanto ao contexto, as quedas ocorreram, predominantemente, durante o dia, fora do ambiente interno institucional, durante a deambulação, em piso de cimento, seco, a partir de tropeços em algo, e quando estavam calçados com chinelos. Os resultados fornecem informações sobre os recursos circunstanciais que podem ser priorizados para a população local, incluindo adequações no ambiente e uso de calçados apropriados, a fim de prevenir as quedas.

Descritores: Idoso; Instituição de Longa Permanência para Idosos; Acidentes por Quedas; Meio Ambiente.

\section{ABSTRACT}

The objective of this study was to identify, among institutionalized elders, the occurrence of falls and the characterization of their context. This descriptive-exploratory study, using a quantitative approach, was performed in five geriatric long-term care facilities in Goiânia (Goiás state). A questionnaire was utilized which addressed information regarding the occurrence of falls in the 2005 to2007 follow-up and their context. The participants were 59 elderly individuals who met the selection criteria. The results showed a $54.2 \%$ prevalence of falls during the studied period. Regarding the context, falls occurred mainly during the day, outdoors, while walking, on dry pavement, due to tripping on/over an object or surface, and while wearing slippers. The results provide information regarding the circumstances surrounding falls that can help to make their prevention a priority for the local population, including addressing inadequacies in the environment and the use of appropriate footwear in order to prevent falls.

Descriptors: Aged; Homes for the Aged; Accidental Falls; Environment.

\section{RESUMEN}

Estudio que apuntó a identificar, en ancianos institucionalizados, la ocurrencia de caídas y caracterizar su contexto. Estudio descriptivo-exploratorio, con abordaje cuantitativo, efectuado en cinco instituciones geriátricas en el municipio de GoiâniaGO. Se aplicó un cuestionario que abordaba informaciones respecto de la ocurrencia de caídas en el período 2005-2007 y su contexto. Participaron del estudio 59 ancianos que atendieron los criterios de inclusión. Los resultados expresaron ocurrencia de $54,2 \%$ de prevalecía de caídas en el período. Respecto al contexto, las caídas sucedieron, predominantemente, durante el día, fuera del ambiente interno institucional, durante la deambulación, en piso de cemento seco, provocadas por tropiezos con alguna cosa, y calzando ojotas. Los resultados brindan informaciones sobre los recursos circunstanciales que pueden priorizarse para la población local, incluyendo adecuaciones ambientales y utilización de calzado apropiado, a efectos de prevenir las caídas.

Descriptores: Anciano; Hogares para Ancianos; Accidentes por Caídas; Ambiente.

\footnotetext{
${ }^{1}$ Fisioterapeuta, Doutora em Ciências da Saúde. Professora Adjunta da Faculdade de Ceilândia da Universidade de Brasília. Brasília, DF, E-mail: ruthlosada@unb.br.

${ }^{2}$ Enfermeira, Doutora em Enfermagem. Professora Titular da Faculdade de Enfermagem da Universidade Federal de Goiás. Goiânia, GO, Brasil. E-mail: $\underline{\text { mbachion@fen.ufg.br. }}$
} 


\section{INTRODUÇÃO}

Queda, segundo o Prevention of Falls Network Europe (ProFaNE) ${ }^{(1)}$, consiste em um evento inesperado de deslocamento involuntário e incontrolável do corpo que faz com que o indivíduo permaneça em nível inferior, por exemplo, sobre mobiliário ou no chão. Estudos indicam que alterações fisiológicas e condições patológicas podem aumentar o risco para quedas(2-3). Fatores ambientais adversos podem criar dificuldades adicionais para os idosos, contrapondo-se a um dos aspectos primordiais para a conquista do envelhecimento ativo: a adoção de um entorno favorável que estimule a mobilidade espacial.

Idosos institucionalizados apresentam, em geral, um histórico de fatores que aumentam a chance de ocorrência de eventos adversos a sua saúde, tais como: sedentarismo, perda da autonomia, ausência de familiares, entre outros, os quais contribuem para o incremento da prevalência de morbidades e comorbidades $^{(4)}$.

Quedas de idosos consistem em grave problema para um país que envelhece aceleradamente, como o Brasil; geram impacto negativo para a saúde pública e são acompanhadas, na maioria das vezes, por consequências como incapacidade, morbidade e morte ${ }^{(5-}$ $6)$.

As quedas consistem em principal causa de lesões, incapacidades, utilização de serviços de cuidados à saúde e, em casos mais extremos, de vida(5). Segundo a Organização Mundial da Saúde (OMS), as quedas são a principal causa de mortalidade por lesão no mundo entre os idosos, respondendo por um terço das mortes por lesões não-intencionais( ${ }^{(6)}$.

Diante dos dados epidemiológicos relacionados às quedas em idosos, sua prevenção constitui-se em desafio para pesquisadores e profissionais de saúde em todo o mundo, especialmente no Brasil, onde o fenômeno do envelhecimento populacional é recente(5).

Recentemente foi realizada uma revisão sistemática com metanálise ${ }^{(7)}$ com o objetivo de determinar a eficácia das intervenções ambientais na redução de quedas em idosos. As intervenções envolveram adaptações e modificações no ambiente físico, além de mudanças no comportamento do idoso durante sua interação com o ambiente. A análise conjunta da amostra final de seis ensaios clínicos $(n=3298)$ demonstrou uma redução de $21 \%$ no risco de quedas (Risco relativo $=0.79$, 95\%; intervalo de confiança $=0.65-0.97$ ). Estes resultados sugerem que as intervenções para prevenção de quedas devem ser abrangentes (e incluir perspectiva ambiental), especialmente as direcionadas aos grupos de alto risco, como os de idosos institucionalizados.

Compreender as circunstâncias em que ocorrem esses incidentes favorece o entendimento do fenômeno por parte da equipe de saúde que assiste esta população. Ademais, poderá nortear medidas de promoção da saúde e prevenção deste agravo. Assim, o objetivo do presente estudo foi identificar, em idosos institucionalizados, a ocorrência de quedas e caracterizar o seu contexto.

\section{MÉTODOS}

Trata-se de um estudo descritivo-exploratório, com abordagem quantitativa, realizado em cinco instituições de longa permanência para idosos no município de Goiânia (GO). A população estudada foi composta por participantes de um projeto maior denominado "Quedas e fatores multidimensionais associados: estudo longitudinal de idosos residentes em instituições de longa permanência em Goiânia (GO)", aprovado pelo Comitê de Ética em Pesquisa da Universidade Federal de Goiás (protocolo 045/2007). No referido estudo foram incluídos idosos que haviam participado de um estudo anterior, intitulado "Quedas em idosos institucionalizados no município de Goiânia", aprovado pelo Comitê de Ética em Pesquisa da Universidade Federal de Goiás (protocolo 032/2004).

Рara melhor visualização, apresentamos um fluxograma. 
Figura 1: Projetos de pesquisa envolvidos na produção de dados do presente estudo.

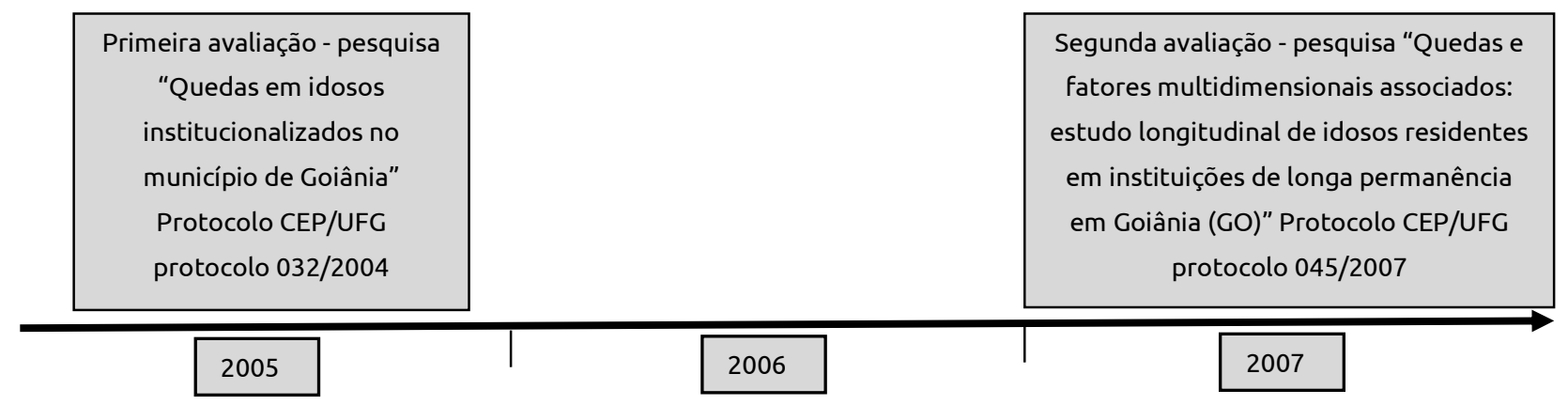

A amostra consistiu de 59 pessoas que se encontravam institucionalizadas. Para a seleção dos participantes foram adotados os seguintes critérios de inclusão na primeira avaliação (2005): ter 60 anos ou mais; morar na instituição há, no mínimo, 36 meses (três anos); deambular, mesmo com auxílio de dispositivo de auxílio à marcha, e ter respondido ao inventário de ocorrência de quedas em 2005 e 2007. Foram excluídos os idosos que apresentavam déficits cognitivos e déficit visual ou auditivo severamente limitante e não compensado por uso de óculos ou aparelho de amplificação sonora, o que dificultaria ou impediria a realização dos testes de avaliação propostos.

Em ambos os projetos de pesquisa os idosos foram esclarecidos sobre a natureza dos estudos, seus objetivos e procedimentos, e consultados quanto ao aceite em participação, de acordo com as recomendações da Resolução 196/96 do Conselho Nacional de Saúde. Após esclarecimento, os idosos foram convidados a participar da pesquisa inicial e da pesquisa de seguimento e, em caso de aceite, o manifestaram mediante assinatura do Termo de Consentimento Livre e Esclarecido (TCLE).

O procedimento de coleta de dados foi realizado pela pesquisadora fisioterapeuta, acompanhada por dois auxiliares de pesquisa (alunos de graduação de fisioterapia) devidamente treinados, no período de maio a agosto de 2007. Os dias das avaliações foram agendados com os responsáveis pelas instituições e os horários para abordagem dos idosos foram escolhidos de acordo com a rotina, local e disponibilidade da pesquisadora e entrevistados. Os idosos foram avaliados em ambientes variados das instituições, mais especificamente nos quartos e áreas externas.

Para a coleta dos dados foi suficiente apenas uma entrevista com cada idoso, com duração de aproximadamente uma hora e meia. Após aceitar e assinar o TCLE, tanto na primeira como na segunda avaliação, cada idoso, sentado em uma cadeira, foi entrevistado por meio de um formulário estruturado. Questionava-se a ocorrência de episódios de quedas nos últimos dois anos e seu contexto (de maio-agosto de 2005 a maio-agosto de 2007).

A investigação do contexto das quedas envolveu: período do dia de ocorrência, lugar (cômodo) onde caiu, atividade que realizava, superfície onde se deu a queda, estado da superfície (molhado/seco), tropeço ou não em obstáculo, tipo de obstáculo e tipo de calçado que usava. A definição de queda considerada neste estudo foi a de qualquer incidente no qual a pessoa venha ao chão ou a um nível inferior, independentemente de sua vontade.

Foram coletados, ainda, no prontuário ou fichas dos idosos, os dados sociodemográficos e, junto ao serviço de enfermagem, checaram-se os episódios de queda.

Empregou-se o software SPSS ${ }^{\circ}$ versão 10.0 , e visando analisar a ocorrência de quedas, quedas recorrentes e seu contexto foi utilizado frequência simples.

\section{RESULTADOS}

A amostra analisada foi composta de $57,6 \%$ de idosos do sexo feminino. A média de idade foi de 78,44 7,8 , prevalecendo a faixa etária de 80 anos ou mais $(47,4 \%)$, vindo, a seguir, a de 70 a 79 anos $(33,8 \%)$ e de 60 a 69 anos (18,8\%).

No seguimento estudado, após a primeira avaliação realizada no ano 2005, dos 59 idosos investigados, 32 (54,2\%) relataram ter sofrido queda, predominando um episódio entre os caidores, com um total de até três episódios. A ocorrência de quedas e suas recorrências encontram-se na Tabela 1. 
Tabela 1: Ocorrência de quedas e quedas recorrentes relatadas

por idosos institucionalizados em Goiânia, GO, 2005-2007.

\begin{tabular}{|c|c|c|c|}
\hline \multicolumn{2}{|c|}{ Ocorrência de quedas } & $\mathbf{N}$ & $\%$ \\
\hline \multirow{3}{*}{$\begin{array}{l}\text { Idosos que caíram } \\
\qquad(n=32)\end{array}$} & Uma vez & 22 & $37,3^{*}$ \\
\hline & Duas vezes & 06 & $10,2^{*}$ \\
\hline & Três vezes & 04 & $6,8^{*}$ \\
\hline \multicolumn{2}{|c|}{ Idosos que não caíram } & 27 & 45,8 \\
\hline \multicolumn{2}{|c|}{ Total } & 59 & 100,0 \\
\hline
\end{tabular}

${ }^{*}$ Percentuais com aproximação.

O quantitativo de ocorrência de quedas no seguimento descrito, 2005-2007, abrangeu três episódios, número máximo relatado pelos entrevistados.

\section{Período do dia e local da queda}

Observando-se a Tabela 2, verifica-se que a ocorrência das quedas predominou nos períodos da manhã e tarde: $1^{\circ}$ episódio ( $50 \%$ manhã; $43,8 \%$ tarde), $2^{\circ}$ episódio ( $40 \%$ manhã; $50 \%$ tarde) e $3^{\circ}$ episódio (50\% manhã; $50 \%$ tarde). Quanto ao local, destacou-se a ocorrência em área externa: $1^{\circ}$ episódio (50\%), $2^{\circ}$ episódio (70\%) e $3^{\circ}$ episódio (75\%).

Tabela 2: Período do dia e local da queda relatados por idosos institucionalizados em Goiânia, GO, 2005-2007.

\begin{tabular}{|c|c|c|c|c|c|c|c|c|c|}
\hline \multirow{3}{*}{ Contexto } & \multicolumn{3}{|c|}{$1^{\circ}$ Episódio } & \multicolumn{3}{|c|}{$2^{\circ}$ Episódio } & \multicolumn{3}{|c|}{$3^{\circ}$ Episódio } \\
\hline & \multirow{2}{*}{ n } & \multirow{2}{*}{$\%$} & IC95\% & \multirow{2}{*}{$n$} & \multirow{2}{*}{$\%$} & IC95\% & \multirow{2}{*}{ n } & \multirow{2}{*}{$\%$} & IC95\% \\
\hline & & & $\%$ & & & $\%$ & & & $\%$ \\
\hline \multicolumn{10}{|l|}{ Período } \\
\hline Manhã & 16 & 50,0 & $31,9-68,1$ & 04 & 40,0 & $12,2-73,8$ & 02 & 50,0 & $6,8-93,2$ \\
\hline Tarde & 14 & 43,8 & $26,4-62,3$ & 05 & 50,0 & $18,7-81,3$ & 02 & 50,0 & $6,8-93,2$ \\
\hline Noite & 01 & 3,1 & $0,1-16,2$ & - & - & - & - & - & - \\
\hline Madrugada & 01 & 3,1 & $0,1-16,2$ & 01 & 10,0 & $0,3-44,5$ & - & - & - \\
\hline \multicolumn{10}{|l|}{ Local } \\
\hline Quarto & - & - & - & 01 & 10,0 & $0,3-44,5$ & - & - & - \\
\hline Corredor & 01 & 3,1 & $0,1-16,2$ & - & - & - & - & - & - \\
\hline Cozinha & 03 & 9,4 & $2,0-25,0$ & - & - & - & - & - & - \\
\hline Banheiro & 03 & 9,4 & $2,0-25,0$ & 01 & 10,0 & $0,3-44,5$ & - & - & - \\
\hline Refeitório & 01 & 3,1 & $0,1-16,2$ & 01 & 10,0 & $0,3-44,5$ & - & - & - \\
\hline Área externa & 16 & 50,0 & $31,9-68,1$ & 07 & 70,0 & $34,8-93,3$ & 03 & 75,0 & $19,4-99,4$ \\
\hline Outros & $08^{(\mathrm{a})}$ & 25,0 & $8,3-57,8$ & - & - & - & $01^{(\mathrm{b})}$ & 25,0 & $0,6-80,6$ \\
\hline Total & 32 & 100,0 & & 10 & 100,0 & & 04 & 100,0 & \\
\hline
\end{tabular}

(a) 01 caiu da cama, 01 no ônibus, 03 fora do ambiente institucional, 03 na rua; (b) 01 na rua.

\section{Atividade realizada}

Quanto às atividades desempenhadas no momento da queda, nos três episódios relatados destacou-se a atividade "estar andando": 65,6\% no primeiro episódio, $50 \%$ no segundo e $75 \%$ no terceiro, conforme Tabela 3. 
Tabela 3: Atividade realizada durante os episódios de queda relatados por idosos institucionalizados em Goiânia, GO, 2005-2007.

\begin{tabular}{|c|c|c|c|c|c|c|c|c|c|}
\hline \multirow{3}{*}{ Contexto } & \multicolumn{3}{|c|}{$1^{\circ}$ Episódio } & \multicolumn{3}{|c|}{$2^{\circ}$ Episódio } & \multicolumn{3}{|c|}{$3^{\circ}$ Episódio } \\
\hline & \multirow{2}{*}{$\mathbf{n}$} & \multirow{2}{*}{$\%$} & IC95\% & \multirow{2}{*}{$\mathbf{n}$} & \multirow{2}{*}{$\%$} & IC95\% & \multirow{2}{*}{$\mathbf{n}$} & \multirow{2}{*}{$\%$} & IC95\% \\
\hline & & & $\%$ & & & $\%$ & & & $\%$ \\
\hline \multicolumn{10}{|l|}{ Atividade realizada } \\
\hline Parado(a), de pé & 03 & 9,4 & $2,0-25,0$ & 04 & 40,0 & $12,2-73,8$ & 01 & 25,0 & $0,6-80,6$ \\
\hline Andando & 21 & 65,6 & $46,8-81,4$ & 05 & 50,0 & $18,7-81,3$ & 03 & 75,0 & $19,4-99,4$ \\
\hline Subindo ou descendo escada/degrau & 04 & 12,5 & $3,5-29,0$ & 01 & 10,0 & $0,3-44,5$ & - & - & - \\
\hline Outra situação & $04^{(c)}$ & 12,5 & $3,5-29,0$ & - & - & - & - & - & - \\
\hline Total & 32 & 100,0 & & 10 & 100,0 & & 04 & 100,0 & \\
\hline
\end{tabular}

(c) 01 assustou-se enquanto dormia, 01 descia da cadeira, 01 em cima da cadeira, 01 tomando banho.

\section{Características do piso e estado da superfície}

Quanto ao tipo de superfície onde ocorreram as quedas, o cimento foi relatado pela maioria nos três episódios consecutivos, 43,8\%, $50 \%$ e $100 \%$, respectivamente. No que diz respeito ao estado da superfície, predominou o estado seco: $75 \%, 70 \%$ e $50 \%$, respectivamente (Tabela 4 ).

Tabela 4: Características do piso e estado da superfície onde ocorreram os episódios de queda relatados por idosos institucionalizados em Goiânia, GO, 2005-2007.

\begin{tabular}{|c|c|c|c|c|c|c|c|c|c|}
\hline \multirow{3}{*}{ Contexto } & \multicolumn{3}{|c|}{$1^{\circ}$ Episódio } & \multicolumn{3}{|c|}{$2^{\circ}$ Episódio } & \multicolumn{3}{|c|}{$3^{\circ}$ Episódio } \\
\hline & \multirow{2}{*}{ n } & \multirow{2}{*}{$\%$} & IC95\% & \multirow{2}{*}{$\mathbf{n}$} & \multirow{2}{*}{$\%$} & \multirow{2}{*}{$\begin{array}{c}\text { IC95\% } \\
\%\end{array}$} & \multirow{2}{*}{ n } & \multirow{2}{*}{$\%$} & IC95\% \\
\hline & & & $\%$ & & & & & & $\%$ \\
\hline \multicolumn{10}{|l|}{ Característica do piso } \\
\hline Cimento & 14 & 43,8 & $26,4-62,3$ & 05 & 50,0 & $18,7-81,3$ & 04 & 100,0 & $100,0-100,0$ \\
\hline Cerâmica/ladrilho/mármore & 10 & 31,3 & $16,1-50,0$ & 02 & 20,0 & $2,5-55,6$ & - & - & - \\
\hline Outro tipo & $08^{(d)}$ & 25,0 & $11,5-43,4$ & $03^{(e)}$ & 30,0 & $6,7-65,2$ & - & - & - \\
\hline \multicolumn{10}{|l|}{ Estado da superfície } \\
\hline Seco & 24 & 75,0 & $56,6-88,5$ & 07 & 70,0 & $34,8-93,3$ & 02 & 50,0 & $6,8-93,2$ \\
\hline Molhado & 08 & 25,0 & $11,5-43,4$ & 03 & 30,0 & $6,7-65,2$ & 02 & 50,0 & $6,8-93,2$ \\
\hline Total & 32 & 100,0 & & 10 & 100,0 & & 04 & 100,0 & \\
\hline
\end{tabular}

(d) 01 na superfície do ônibus, 01 terra/mato, 06 terra; (e) 03 terra.

\section{Tropeços e calçados}

A porcentagem de tropeços aumentou ao serem comparados os três episódios e observou-se que o caidor crônico apresentou maior ocorrência deste incidente (75\%), indicando, possivelmente, maior fragilidade ao vencer obstáculos.

No primeiro episódio de queda, o local do tropeço ocorreu majoritariamente em objeto/obstáculo (54,5\%); no segundo episódio nos chinelos $(66,7 \%)$ e no terceiro episódio houve igual distribuição em chinelos, degrau/escada e objeto/obstáculo (33,3\%), com exceção do tropeço nos próprios pés, que não ocorreu neste último.

Em todos os episódios de queda, a maioria dos entrevistados fazia uso de chinelos: $43,8 \%$, $80 \%$ e $75 \%$, consecutivamente. Todas as características envolvendo os tropeços e calçados encontram-se detalhadas na Tabela 
Tabela 5: Tropeços e calçados envolvidos durante os episódios de queda relatados por idosos institucionalizados em Goiânia, GO, 2005-2007.

\begin{tabular}{|c|c|c|c|c|c|c|c|c|c|}
\hline \multirow{3}{*}{ Contexto } & \multicolumn{3}{|c|}{$1^{\circ}$ Episódio } & \multicolumn{3}{|c|}{$2^{\circ}$ Episódio } & \multicolumn{3}{|c|}{$3^{\circ}$ Episódio } \\
\hline & \multirow{2}{*}{$\mathbf{n}$} & \multirow{2}{*}{$\%$} & IC 95\% & \multirow{2}{*}{$n$} & \multirow{2}{*}{$\%$} & IC 95\% & \multirow{2}{*}{$\mathbf{n}$} & \multirow{2}{*}{$\%$} & \multirow{2}{*}{$\begin{array}{c}\text { IC 95\% } \\
\%\end{array}$} \\
\hline & & & $\%$ & & & $\%$ & & & \\
\hline \multicolumn{10}{|l|}{ Tropeço } \\
\hline Sim & 11 & 34,4 & $18,6-53,2$ & 03 & 30,0 & $6,7-65,2$ & 03 & 75,0 & $19,4-99,4$ \\
\hline Não & 21 & 65,6 & $46,8-81,4$ & 07 & 70,0 & $34,8-93,3$ & 01 & 25,0 & $0,6-80,6$ \\
\hline \multicolumn{10}{|l|}{ Local /tropeço } \\
\hline Chinelos & 02 & 18,2 & $2,3-51,8$ & 02 & 66,7 & $9,4-99,2$ & 01 & 33,3 & $0,8-90,6$ \\
\hline Degrau/calçada & 01 & 9,1 & $0,2-41,3$ & - & - & - & 01 & 33,3 & $0,8-90,6$ \\
\hline Objeto/obstáculo & 06 & 54,5 & $23,4-83,3$ & 01 & 33,3 & $0,8-90,6$ & 01 & 33,3 & $0,8-90,6$ \\
\hline Próprios pés & 02 & 18,2 & $2,3-51,8$ & - & - & - & - & - & - \\
\hline \multicolumn{10}{|l|}{ Calçado } \\
\hline Chinelo & 14 & 43,8 & $26,4-62,3$ & 08 & 80,0 & $44,4-97,5$ & 03 & 75,0 & $19,4-99,4$ \\
\hline Sapato com sola de borracha & 08 & 25,0 & $11,5-43,4$ & - & - & - & - & - & - \\
\hline Outro & $10^{(f)}$ & 31,3 & $16,1-50,0$ & $02^{(g)}$ & 20,0 & $2,5-55,6$ & $01^{(\mathrm{h})}$ & 25,0 & $0,6-80,6$ \\
\hline Total & 32 & 100,0 & & 10 & 100,0 & & 04 & 100,0 & \\
\hline
\end{tabular}

(f) 01 tênis, 02 sapatilhas, 01 sandália de borracha, 02 sandália com salto, 04 descalço; (9) 01 sandália de borracha, 01 tênis; ${ }^{(h)} 01$ sandália de borracha.

\section{DISCUSSÃO}

Quanto ao período do dia em que ocorreram as quedas dos idosos, o período diurno, predominante nos achados do presente estudo, corrobora os resultados de outras pesquisas $^{(3,8-10)}$. Os idosos apresentam o hábito de acordar mais cedo e iniciam suas atividades e tarefas diárias pela manhã; estão, assim, mais predispostos a quedas neste período.

Este resultado tem implicação potencial para o serviço de enfermagem oferecido em instituições onde os idosos geralmente são acompanhados por cuidadores formais e informais, sob a supervisão direta do enfermeiro. Cabe, assim, a estes profissionais, juntamente com a equipe multiprofissional, maior cuidado e estratégias de prevenção nos horários mais prováveis de ocorrência de quedas.

As quedas que ocorrem a noite ou durante a madrugada podem estar relacionadas ao fato de que nestes períodos os idosos se deslocam para beber água ou para ir ao banheiro e, muitas vezes, deparam-se com condições ambientais desfavoráveis, como a iluminação inadequada. Sugere-se, portanto, que interruptores de luminosidade fiquem ao alcance dos idosos, assim como fonte de água. Horários de medicação também deveriam ser ajustados para evitar picos de produção de diurese no período noturno. Tais medidas poderiam evitar deslocamentos nesse intervalo.
Estudos referem que o local de queda mais comum é o próprio ambiente da instituição ou a própria casa, especialmente os quartos ${ }^{(11-12)}$. A maior ocorrência de quedas no interior da moradia do idoso pode ser justificada pela fragilidade e limitação funcional que pode ocorrer nas pessoas desta faixa etária.

Nas Instituições de Longa Permanência para Idosos (ILPI) os moradores em situação de fragilidade poderiam ser acomodados em quartos nos quais fossem acompanhados por cuidadores formais ou informais no período noturno; assim, sua situação de maior vulnerabilidade seria protegida.

Idosos saudáveis tendem a cair fora de casa durante atividades instrumentais, enquanto idosos frágeis tendem a cair em casa durante atividades cotidianas, sem grandes exigências sobre o equilíbrio(13). Desta forma, pode-se abstrair que os idosos institucionalizados participantes deste estudo são menos frágeis e mais independentes nas atividades cotidianas. Os maiores riscos de queda são enfrentados fora do ambiente interno institucional, onde existem maiores desafios, como: solo irregular, presença de meio-fio (degrau) e superfícies molhadas.

Há um consenso na literatura de que o idoso cai durante a deambulação(8-9) e atividades rotineiras(14). A minoria de quedas seria decorrente de atividades arriscadas, como subir em cadeiras ou praticar esportes $^{(15)}$. 
O declínio da força e da massa muscular, assim como a diminuição da flexibilidade, causados pelo envelhecimento, podem ser fatores limitantes para o desempenho e manutenção da marcha em idosos ${ }^{(16)}$. Deste modo, torna-se importante planejar, implementar e avaliar medidas preventivas para a manutenção do ato da deambulação de forma segura, por mais tempo e com a participação e envolvimento da equipe multidisciplinar.

Há evidências de que a implementação de programas de exercícios físicos podem melhorar a força muscular, a marcha e o equilíbrio entre as pessoas idosas e, consequentemente, diminuir o número de quedas ${ }^{(16)}$. Outras medidas importantes para assegurar uma deambulação segura são: a avaliação e indicação, quando necessário, de dispositivos de auxílio à marcha, dispositivos para correção visual e utilização de calçados adequados.

O piso escorregadio, molhado e irregular é reconhecido consensualmente pela literatura como fator predisponente a quedas ${ }^{(17)}$. O cimento e o asfalto são as superfícies empregadas majoritariamente nos ambientes externos à instituição, local onde aconteceu a maioria das quedas dos idosos investigados no presente estudo. Neste caso, outros fatores (não relacionados diretamente à superfície) podem ter sido determinantes para a ocorrência dos eventos, como os obstáculos ambientais, o que requer outras investigações.

A contribuição do calçado para a instabilidade postural e quedas em pessoas idosas tem recebido relativamente pouca atenção na literatura, apesar de uma grande proporção de quedas ocorrer quando as pessoas mais velhas estão caminhando ou transpondo obstáculos comuns, tais como calçadas e escadas ${ }^{(18)}$.

O uso de calçados inapropriados foi associado a quedas em estudos realizados por outros autores ${ }^{(10)}$. Calçados inadequados alteram o feedback somatossensorial do tornozelo/pé, modificando as condições de atrito com o solo, e influenciam, assim, na estabilidade postural e no consequente risco de escorregões, tropeços e quedas ${ }^{(19)}$.

A moda influenciou fortemente o desenho do calçado ao longo dos tempos, comprometendo o funcionamento natural do pé e contrariando suas funções principais, que seriam proteger o pé e facilitar a deambulação. Saltos altos, saltos estreitos, solas escorregadias, solas moles e colarinhos do calcanhar sem suporte são características de calçado prejudiciais ${ }^{(19)}$.

A associação positiva entre o uso de calçados acolchoados atléticos e risco aumentado de quedas foi identificada em estudo(20) com 106 pessoas idosas morando em comunidades. Isso nos faz pensar que os usuários desse estilo de calçado sejam idosos ativos e que o comportamento de risco (realizar atividade física) e/ou outros fatores podem ter gerado a disposição a quedas, uma vez que este evento é um fenômeno multifatorial.

Como nas ILPI os idosos recebem acompanhamento de enfermagem 24 horas, essa equipe tem maiores condições de intervir junto a população idosa no sentido de promover o uso de calçados mais adequados. Nem sempre o tipo de calçado é uma escolha do idoso, em todas as ILPI, e nesse caso, a equipe multiprofissional de saúde deve se engajar, junto aos gestores, para o fornecimento de calçados adequados.

As quedas consistem em eventos adversos e seu registro é extremamente importante para a organização, planejamento e execução do processo de trabalho em saúde, especialmente no ambiente institucional. Devido ao potencial dano ao idoso que este acidente pode gerar, a comunicação da ocorrência e seu contexto à equipe de gerenciamento em saúde permitirá a adoção de mecanismos adequados de prevenção(21).

No presente estudo, pode ter ocorrido subnotificação da ocorrência de quedas e seu contexto, pois, do longo período (dois anos) em que foi solicitada do idoso a recordação do incidente, mesmo com o cuidado de se checar os episódios com o serviço de enfermagem, a quantidade máxima relatada não ultrapassou três por pessoa. Para a classificação de idosos em caidores e não-caidores, o padrão ouro atual é o registro sistemático do evento, sempre que ele ocorrer, e um sistema de notificação com um mínimo de relatórios mensais(22). Neste sentido, destaca-se a importância da documentação adequada de enfermagem, que se constitui a categoria profissional com acesso aos idosos por mais tempo nas ILPI.

\section{CONCLUSÃO}

No ambiente de instituições de longa permanência para idosos, independente da existência ou nível de fragilidade dessas pessoas, o que já implicaria 
considerável risco para a ocorrência de queda, coexistem fatores de risco de outras naturezas para a incidência deste evento adverso, como os ambientais, os temporais e de vestuário.

Os resultados apresentados mostram que os objetivos propostos inicialmente foram contemplados e levam à constatação de que a prevenção a quedas deve envolver uma abordagem multifatorial, e a interação do idoso com o meio ambiente deve ser observada e planejada pelos serviços oferecidos nesses locais.

Para manter a capacidade funcional dos idosos, mais especificamente as funções relacionadas à capacidade de deambulação, é importante o favorecer a movimentação e o deslocamento significativo, ou seja, andar distâncias maiores que as possibilitadas apenas no interior das ILPI. Essa independência pode favorecer os processos de socialização, tão importantes para o envelhecimento saudável e a qualidade de vida.

\section{REFERÊNCIAS}

1. Lamb SE, Jorstad-Stein EC, Hauer K, Becker C. Development of a common outcome data set for fall injury prevention trials: the Prevention of Falls Network Europe consensus. J Am Geriatric Soc. 2005;53:1618-22.

2. French DD, Werner DC, Campbell RR, Powell-Cope GM, Nelson AL, Rubenstein LZ et al. (2007). A multivariate fall risk assessment model for VHA nursing homes using the minimum data set. JAMDA. 2007;8(2):115-22.

3. Hill EE, Nguyen TH, Shaha M, Wenzel JA, DeForge BR, Spellbring AM. Person-Environment Interactions Contributing to Nursing Home Resident Falls. Res Gerontol Nurs. 2009;2(4):287-96.

4. Lamb SE, Jorstad-Stein EC, Hauer K, Becker C. Development of a common outcome data set for fall injury prevention trials: the Prevention of Falls Network Europe consensus. J Am Geriatric Soc. 2005;53:1618-22.

5. Planek TW. Falls among the elderly. J Safety Res. 2011;42(6):415.

6. World Health Organization (WHO) (2010). WHO Statistical Information System (WHOSIS). Available from: http://whalibdoc.who.int/publications/2010/9789241599375_e ng.pdf

7. Clemson L, Mackenzie L, Ballinger C, Close JC, Cumming RG. Environmental interventions to prevent falls in communitydwelling older people: a meta-analysis of randomized trials. J Aging Health. 2008;20(8):954-71.

8. Santos MLC, Andrade MC. Incidência de quedas relacionada aos fatores de risco em idosos institucionalizados. Rev Baiana Saúde Pública. 2005;29(1):57-68.

9. Nascimento FA, Vareschi AP, Alfieri FM. Prevalência de quedas, fatores associados e mobilidade funcional em idosos institucionalizados. ACM Arq Catarin Med. 2008;37(2):7-12. 10. Costa AGSC, Souza RC, Vitor AF, Araujo TL. Acidentes por quedas em um grupo específico de idosos. Rev. Eletr. Enf. [Internet]. 2011 [cited 2012 abr 27];13(3):395-404. Available from: http://www.fen.ufg.br/revista/v13/n3/v13n3a04.htm.
Com o objetivo de evidenciar a aplicabilidade dos resultados desta pesquisa, reforçamos algumas recomendações que visam o controle e prevenção de quedas em idosos institucionalizados, como a adoção de algumas medidas especialmente direcionadas ao ambiente e registro de quedas, tais como: instituir nas ILPI um boletim de quedas que relate data e hora de ocorrência do episódio, local, atividade desempenhada, sintomas, tipos e características de calçados utilizados; reconhecer os idosos que têm maior predisposição a quedas, identificando os fatores de risco extrínsecoambientais, assim como os intrínsecos a que estão expostos; possibilitar o uso de calçados apropriados; instalar medidas de segurança ambientais, tais como: rampas, piso antiderrapante, iluminação e móveis adequados, corrimãos nos locais de maior risco; implementação de estratégias de revitalização urbana, especialmente nas calçadas e meios-fios, em prol do deslocamento seguro do idoso no ambiente urbano.

11. Gonçalves LG, Vieira ST, Siqueira FV, Hallal PC. Prevalência de quedas em idosos asilados do município de Rio Grande, RS. Rev. Saúde Pública. 2008;42(5):938-45.

12. Siqueira FV, Facchini LA, Piccini RX, Tomasi E, Thumé E, Silveira DS et al . Prevalência de quedas em idosos e fatores associados. Rev Saúde Pública. 2007;41(5):749-56.

13. Kelsey JL, Procter-Gray E, Berry SD, Hannan MT, Kiel DP, Lipsitz LA, Li W. Reevaluating the Implications of Recurrent Falls in Older Adults: Location Changes the Inference. JAGS.

2012;60:517-24.

14. Silva TM, Nakatani AYK, Souza ACS, Lima MCS. A vulnerabilidade do idoso para as quedas: análise dos incidentes críticos. Rev. Eletr. Enf. [Internet]. 2007 [cited 2012 abr 27];9(1):64-78. Available from:

http://www.fen.ufg.br/revista/v9/n1/v9n1a05.htm

15. Fabrício SCC, Rodrigues RAP, Costa Júnior ML. Causas e conseqüências de quedas de idosos atendidos em hospital público. Revista de Saúde Pública. 2004;38(1): 93-9.

16. Sherrington C, Tiedemann A, Fairhall N, Close JC, Lord SR. Exercise to prevent falls in older adults: an updated metaanalysis and best practice recommendations. N S W Public Health Bull. 2011;22(3-4):78-83.

17. Ramos CV, Santos SSC, Barlem ELD, Pelzer MT. Quedas em idosos de dois serviços de pronto atendimento do Rio Grande do Sul. Rev. Eletr. Enf. [Internet]. 2011 [cited 2012 abr 27];13(4):703-13. Available from:

http://www.fen.ufg.br/revista/v13/n4/v13n4a15.htm. 18. Norton R, Campbell AJ, Lee-Joe T, Robinson E, Butler M. Circumstances of falls resulting in hip fractures among older people. J Am Geriatr Soc. 1997;45(9):1108-12.

19. Menant JC, Steele JR, Menz HB, Munro BJ, Lord SR. Optimizing footwear for older people at risk of falls. J Rehabil Res Dev. 2008;45:1167-81.

20. Frey CC, Kubasak M. Faulty footwear contributes to why seniors fall. Biomechanics. 1998;5:45-7.

21. Paiva MCMS, Paiva SAR, Berti HW, Campana AO.

Caracterização das quedas de pacientes segundo notificação em 
boletins de eventos adversos. Rev Esc Enferm USP.

2010;44(1):134-8.

22. Delbaere K, Close JC, Kochan NA, Sachdev PS, Lord SR. Good

memory as a predictor of falls: fact or artefact. Age Ageing.

2012;41(3):404-8.

Artigo recebido em 09/07/2011.

Aprovado para publicação em 02/04/2012.

Artigo publicado em 30/09/2012. 